\title{
Systematic Review and Meta-Analysis of the Use of Hyaluronic Acid Injections to Restore Interproximal Papillae
}

\author{
Arturo Sanchez-Perez ${ }^{1, * \mathbb{D}}$, Tania Rosa Vela-García ${ }^{1}$, Bibiana Mateos-Moreno ${ }^{1}$, Alfonso Jornet-García ${ }^{1}$ \\ and Carlos Navarro-Cuellar ${ }^{2}$ (D) \\ 1 Medicine and Dentistry Faculty, Department of Periodontology, Murcia University, 30008 Murcia, Spain; \\ taniarosa.velag@um.es (T.R.V.-G.); bibimateos@um.es (B.M.-M.); alfonsofelipe.jornet@um.es (A.J.-G.) \\ 2 Department of Surgery, Complutense University, 28040 Madrid, Spain; cnavarrocuellar@gmail.com \\ * Correspondence: arturosa@um.es
}

check for updates

Citation: Sanchez-Perez, A.; Vela-García, T.R.; Mateos-Moreno, B.; Jornet-García, A.; Navarro-Cuellar, C. Systematic Review and

Meta-Analysis of the Use of Hyaluronic Acid Injections to Restore Interproximal Papillae. Appl. Sci. 2021, 11, 10572. https://doi.org/ 10.3390/app112210572

Academic Editor: Gabi Chaushu

Received: 26 September 2021

Accepted: 8 November 2021

Published: 10 November 2021

Publisher's Note: MDPI stays neutral with regard to jurisdictional claims in published maps and institutional affiliations.

Copyright: (c) 2021 by the authors. Licensee MDPI, Basel, Switzerland. This article is an open access article distributed under the terms and conditions of the Creative Commons Attribution (CC BY) license (https:// creativecommons.org/licenses/by/ $4.0 /)$.

\begin{abstract}
The absence of interdental papillae leads to the appearance of black triangles. For most patients, the appearance of these triangles is an important reason for concern and affects their social relationships. Multiple reconstruction techniques have been developed with different degrees of success and predictability. The main aim of this study was to evaluate the efficiency of hyaluronic acid (HA) injected into interproximal papillae 6 months after injection and to perform a systematic review and meta-analysis. After a systematic review, seven articles were selected: two clinical randomised controlled trials and five clinical trials. In total, 127 patients with a deficiency in upper papillae in the front of the maxilla and jaw were included in the study. The height variable was evaluated (mm) 6 months after HA injection. In total, 166 interproximal papillae were studied. Four articles showed an important difference in favour of intervention. The total result regarding in the injection of HA was favourable with an approximate average filling of $0.47 \mathrm{~mm}$ in the height of the interdental papilla. The injection of HA for the reconstruction of deficient papillae in the region of the upper and lower maxilla was a possible option treatment strategy in mild black triangles.
\end{abstract}

Keywords: aesthetics; gingiva; hyaluronic acid; injections; surgical procedures; minimally invasive

\section{Introduction}

The interdental papilla is a soft tissue found in the cervical area of the free gingiva, extending from the interproximal alveolar crest to the point of contact [1].

The loss of the interproximal papilla has both functional and aesthetic effects, which have been the subject of numerous studies [2-4].

The cause of the loss of the papilla is multi-factorial; also, the loss of the papilla results in the appearance of so-called "black triangles".

Reconstruction of the interdental papilla has been approached from various techniques. Most of them include surgical techniques with not always predictable results. The minimally invasive HA approach has the potential advantage of being effective, predictable, and minimally invasive with only slight injury [5]. Hence, most efforts have focused on conservation techniques with the main limiting factor being the delicate vascularity of the papilla [6-8].

The importance of the subject lies in knowing the fields of application of HA in clinical terms, its indications and its limitations, for which we reviewed the existing literature in order to provide recommendations based on previously published studies.

Hyaluronic acid (HA) was discovered by Karl Meyer in 1934 and his assistant John Palmer, who isolated the chemical from the vitreous humour of cow eyes.

HA is a non-sulphated glycosaminoglycan consisting of disaccharide units (GAGs), D-glucuronic acid, and N-acetyl glucosamine (NAcGlu) bound by heparin, heparan, chondroitin, and dermatan sulphate. 
The use of hyaluronic acid has been of particular interest as a volumetric filler to restore aesthetics. Therefore, it is highly possible to use HA in dentistry. One of the applications of HA lies in the filling and reconstruction of interdental papilla [9].

The objective of our study was to review the literature of the last 12 years regarding the use of hyaluronic acid in the treatment of interproximal black triangles by rating the available evidence.

\section{Material and Methods}

\subsection{Study Design}

A bibliographic search was carried out with the intention of conducting a metaanalysis based on a systematic review of the literature of the articles published between January 2009 and September 2021.

\subsection{Search Strategy}

The focused question was structured according to the PICO format (target population, intervention to be carried out, comparison, outcomes, and studies) (Table 1 and Figure 1).

Table 1. Parameters used for PICO ( $\mathrm{P}-$ patient, problem or population. I-intervention. C comparison, control, or comparator. O—outcome(s)).

\begin{tabular}{cc}
\hline & PICO Process \\
\hline Population & Patients with interproximal papilla deficiency \\
\hline Intervention & Hyaluronic acid injection \\
\hline Comparison & Treatment with saline serum/placebo/or control prior to the treatment \\
\hline Outcome & Height of the papilla in $\mathrm{mm}$ \\
\hline
\end{tabular}

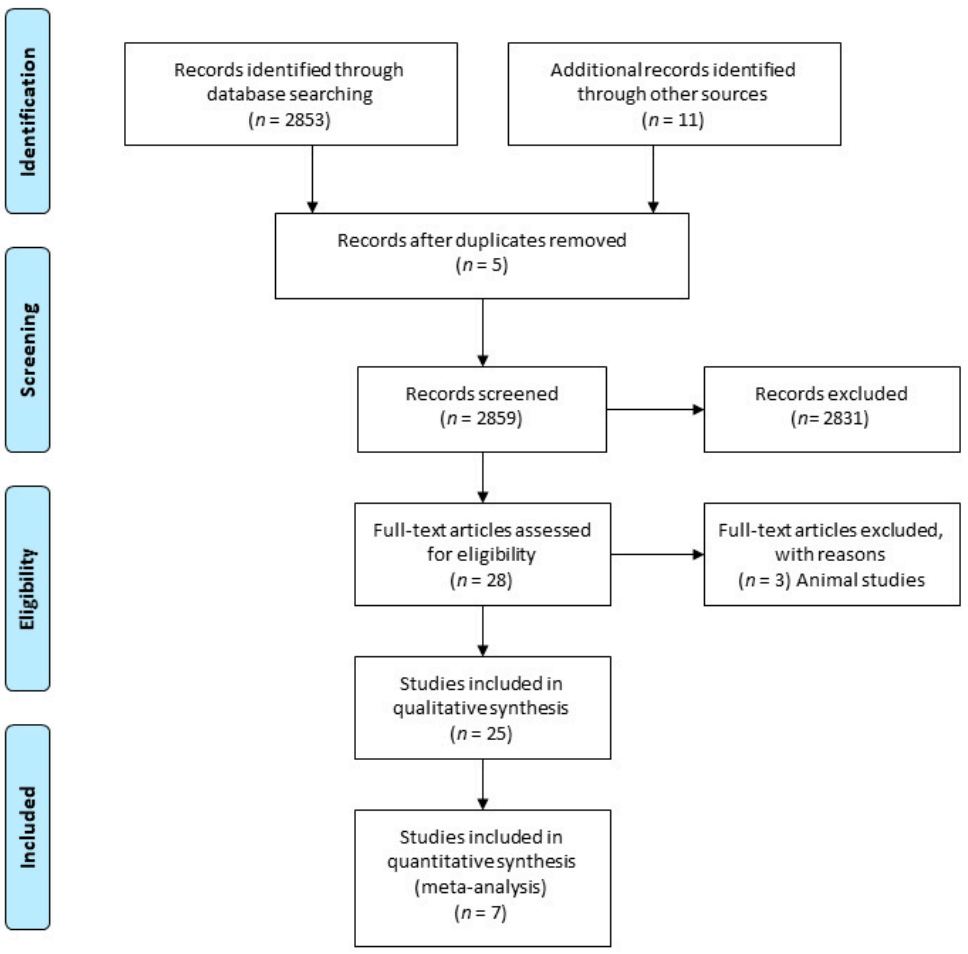

Figure 1. Flowchart of the process of searching for and selecting articles in databases.

Several electronic databases were used, including Scielo, Medline-PubMed, Google Academic, ScienceDirect, Dialnet, as well as journals from the Peruvian Association of Periodontics and Osteointegration, the Spanish Society of Periodontics and Osteointegra- 
tion, and other research journals. The following keywords were used: "filler", "gingival increase", "hyaluronic acid", "hyaluronan", "papilla augmentation", "dental papilla", and "papillary remodelling".

\subsection{Selection of Studies}

The titles and summaries of the studies included in this systematic review were selected. Then, the validity of the studies was assessed according to the score obtained by Jadad, in which seven questions were used to evaluate the validity in a clinical trial. The score ranged from 0 to 5 ; the higher the score was, the better the methodological quality of the clinical trial being evaluated. In addition, the PRISMA guidelines for systematic review verification and meta-analysis were followed [10].

The search was limited to humans with loss of the interdental papilla with no active periodontal disease. The search was not restricted to any particular language. The time period of the studies was limited to the last 12 years.

\subsection{Inclusion Criteria}

The inclusion criteria taken into account for the meta-analysis were as follows: series of cases of at least 10 patients, at least 6 months of follow-up, papilla measurement in mm, adequate hygienic control, and no active periodontal disease.

\subsection{Exclusion Criteria}

Exclusion criteria were as follows: no baseline data; loss of more than $15 \%$ of patients during follow-up; Jemt papilla index of 3-4; non-translatable language and inadequate data; such as no measurement in $\mathrm{mm}$; no measurement in $\mathrm{mm}^{2}$; or lack of sample definition, as well as replication of articles.

\section{Results}

In this systematic review on the use of HA in interdental papilla reconstruction, a total of 127 patients were included, with a total of 244 papillae, all of which were in the anterior upper and lower jaw region.

A total of seven articles with two randomised control trials and five clinical trials (case series) were included to evaluate the effect of HA injection. Singh's article provided three independent comparisons [11] (Figure 2).

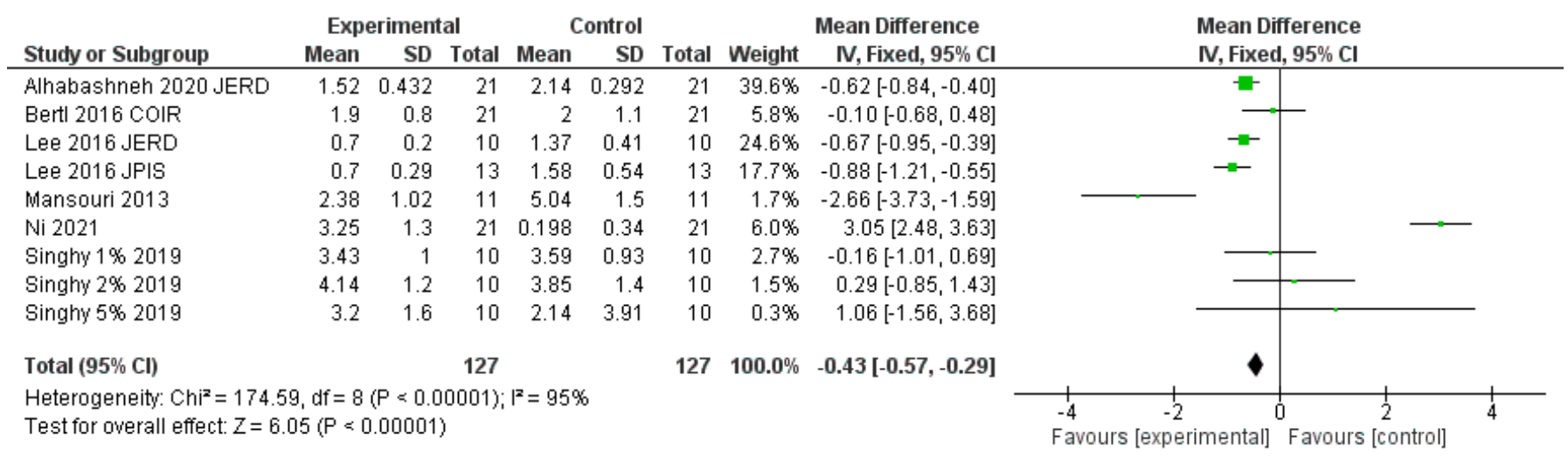

Figure 2. Forest plot reconstruction of interdental papilla deficiency with HA. Height values (mm) indicate a favourable result of HA injection.

The study by Bertl et al. [12] provided non-significant results, a very wide standard deviation and a small mean, reflecting a large population dispersion. The study of Lee et al. [13] was the only one that showed a significant difference after the intervention, as reflected in its weight of the study with $24.6 \%$; the standard deviation, along with the mean, indicated more accurate variability. 
The second study by Lee et al. [14] was also significant but had less weight than the previous study $(17.7 \%)$, as its population dispersion was wider. The study by Mansouri et al. [15] had a very wide dispersion regarding measurements, which represented a wide dispersion of the results; the same was true for all the studies by Singh and Vandana [11].

The greater weight in this review was obtained by the study of Alhabashnaeh [16] with a $39.6 \%$. Even more, it had the lowest dispersion. The study by Ni [17] has a high sample size but with a large amplitude, which reduced its weight in this review by up to $6 \%$.

The overall result was significant in favour of HA injection, with a maximum interdental papilla height of approximately $0.43 \mathrm{~mm}$. Heterogeneity was high at $\mathrm{I}^{2}=95 \%$. There was no statistical homogeneity.

All estimates appeared to be affected by publication bias according to the funnel plot, which shows an asymmetric funnel, i.e., clear publication bias, meaning that more studies about HA for interproximal papilla reconstruction are needed (Figure 3).

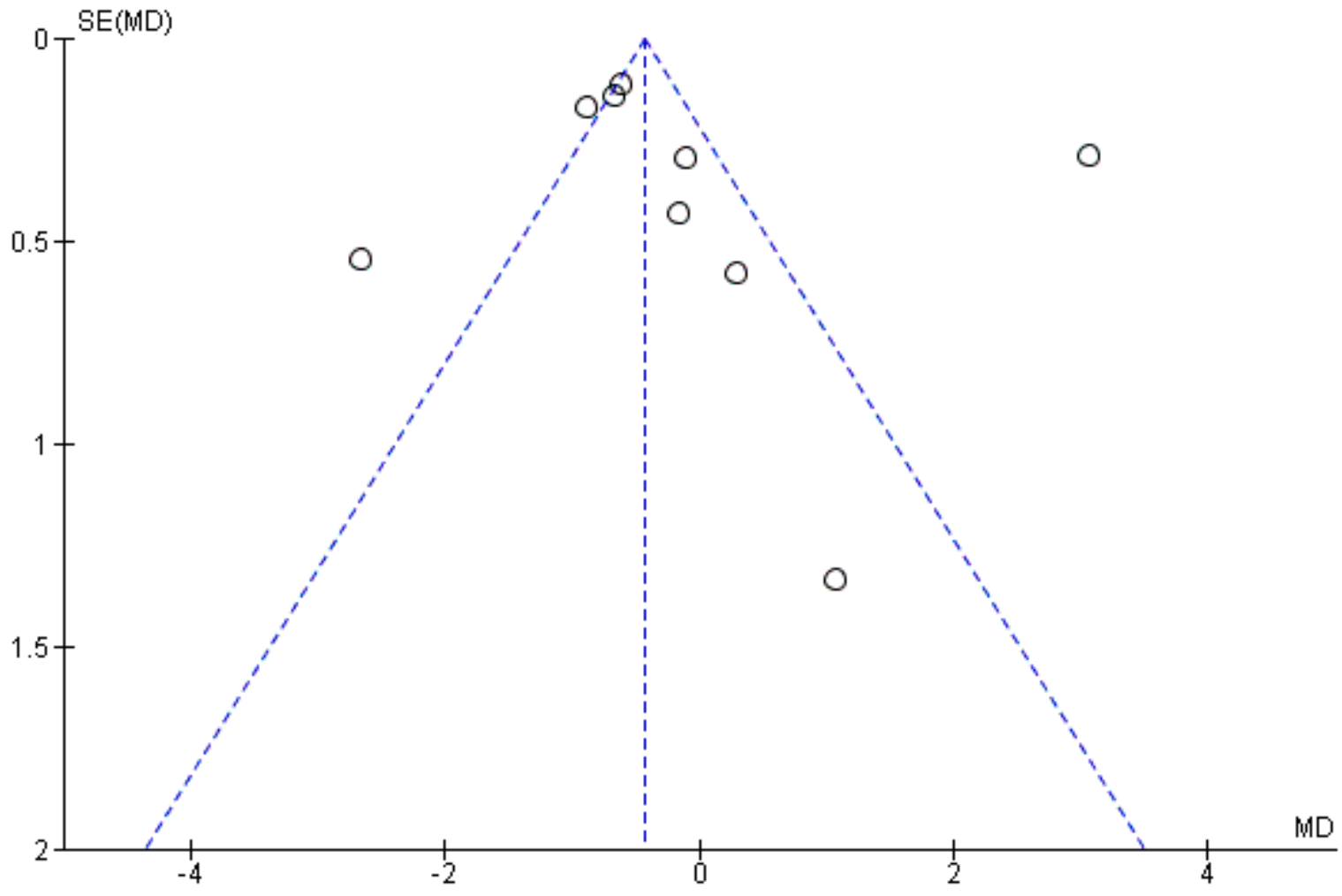

Figure 3. Funnel plot.

The ROBIN I non-randomised risk of bias assessment was used to assess the risk of bias [18]. All studies had a degree of bias risk (Table 2).

One of the randomised clinical trials (RCTs) present in this study was the article by Bertl et al. [12], which presented an unclear risk of bias. This study ended before completion due to the absence of partial results.

The other one RCT was the article of Ni who used physiological saline solution as control, with paradoxically no statistically significant differences.

"To assess the validity of the review, the Kappa coefficient was used. Kappa is a measure of agreement proposed by Cohen in 1960, which is based on comparing the agreement observed in a set of data, with respect to what could occur by mere chance. The agreement obtained in our study was $0.824 . "$ 
Table 2. Assessing risk of bias in included studies. All studies have moderate to high risk of bias. Low risk of bias $=$ green $(+)$, unclear risk of bias = yellow $(i ?)$, high risk of bias $=$ red $(-)$.

\begin{tabular}{|c|c|c|c|c|c|c|c|c|c|}
\hline & 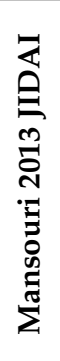 & 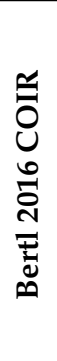 & 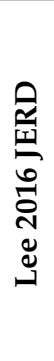 & 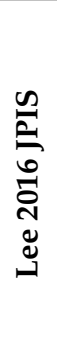 & 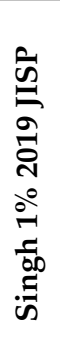 & 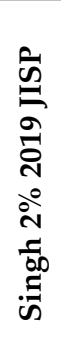 & 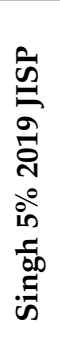 & 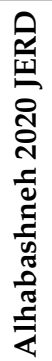 & 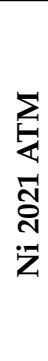 \\
\hline Confusion Bias & - & + & + & + & - & - & - & $?$ & $?$ \\
\hline Selection Bias & + & + & + & + & - & - & - & - & ¿? \\
\hline Classification Bias & - & + & - & $?$ & $?$ & $?$ & $?$ & $?$ & $?$ \\
\hline $\begin{array}{c}\text { Intervention? } \\
\text { Intervention Bias }\end{array}$ & - & $?$ & $?$ & + & + & + & + & + & + \\
\hline Lost Data Bias & + & $?$ & - & $?$ & - & - & - & - & - \\
\hline Measurement Bias & $?$ & + & - & + & - & - & - & + & + \\
\hline Results Bias & + & - & $?$ & + & $?$ & $?$ & $?$ & + & + \\
\hline
\end{tabular}

\section{Discussion}

The prevalence of black triangles is considered to be between $15 \%$ and $41.9 \%$ in the general population depending on the dental procedure performed and the age of the patient $[19,20]$.

Reconstruction of the interdental papilla has been approached from various reconstruction techniques. Most of them include surgical techniques with not always predictable results. The minimally invasive HA approach has the potential advantage of being effective, predictable, and minimally invasive with only slight injury as compared to surgical treatments. In addition, it has few side effects. Although, its application is limited to small defects.

This systematic review and meta-analysis were based on the application of HA to treat the presence of black triangles. A total of 166 papillae in 126 patients were included in the evaluated studies.

The study by Bertl et al. [12] included 13 papillae adjacent to implants in 21 patients (12 women and 9 men) who were injected with HA. The manufacturer's recommended technique was used in the study (Hyadent Barrier Gel, Elaf Medical Supplies, Zagreb, Croatia). Thus, $0.36 \mathrm{~mL}$ of HA were injected, of which $5.76 \mathrm{mg}$ were crosslinked HA and $0.72 \mathrm{mg}$ were non-crosslinked HA.

The average papilla defect at the beginning of the study was $2.0 \pm 1.1$. At the end of the study ( 6 months later), the average was $1.9 \pm 0.8$. This difference was not statistically significant.

In the first study by Lee et al. [13], 10 patients (four men and six women) were studied and injected with HA (a total of 43 papillae adjacent to natural teeth). For the initial examination, papilla defects with cut-off values of $0.24 \mathrm{~mm}^{2}$ for area, $1.0 \mathrm{~mm}$ in height, and $0.5 \mathrm{~mm}$ for width were chosen.

In this study, a total of $0.01 \mathrm{cc}$ was injected (Teosyal Puresense Global Action, Teoxane, Geneva, Switzerland), of which $0.25 \mathrm{mg}$ consisted of crosslinked hyaluronic acid. A maximum of five sessions were performed or sessions were continued until black triangles were no longer visible. The initial mean area was $1.37 \pm 0.41 \mathrm{~mm}$, which was reduced to $0.70 \pm 0.20 \mathrm{~mm}$ at 6 months. Their results represent a decrease in the percentage of area of the black triangle.

The results show 100\% total reconstruction at 29 sites and a partial reconstruction of the remaining 14 sites, with percentages between 39\% and 96\%. Taking the data as a whole, an average reconstruction rate of $92.55 \%$ was achieved following an average number of 3.42 injections. Of note was the $100 \%$ interdental papilla reconstruction rate in most cases 
for initial values of up to $0.25 \mathrm{~mm}^{2}$ area and a height of up to $1 \mathrm{~mm}$. However, when the area exceeded $0.25 \mathrm{~mm}^{2}$ and the height was $1 \mathrm{~mm}$, the results showed a statistically significant negative correlation in relation to the rate of reconstruction.

An interesting finding was that the distance between the point of contact and the bone crest had to be less than $6 \mathrm{~mm}$ for optimal results to be achieved, a result consistent with those obtained by other authors [21-25].

In the second study by Lee et al. [14], 13 patients (six men and seven women) with a total of 21 papillae from the anterior maxilla region were included. Only papillae that had adjacent teeth were included.

In each session, a single dose per 0.002 cc HA (Teosyal Puresense Global Action, Teoxane, Geneva, Switzerland) was used with a maximum of five applications. The time interval between applications was 3 weeks (or until black triangles were no longer visible). The total injected dose was $0.01 \mathrm{cc}$, of which $0.25 \mathrm{mg}$ was crosslinked HA. The initial mean height in mm was $1.58 \pm 0.54$, while at 6 months, the mean height of the papillae was $0.70 \pm 0.29$, which was a statistically significant difference with appreciable clinical effects.

The positive effect of HA on interdental papilla reconstruction was also demonstrated in the study by Singh and Vandana [11] carried out in the anterior region of the maxilla and mandible in 10 patients (two men and eight women). In total, the authors studied 35 papillae.

The selected patients were classified into three groups depending on the concentration of $\mathrm{HA}$ being injected ( $1 \% \mathrm{HA}$ in group 1, $2 \% \mathrm{HA}$ in group 2, and $5 \% \mathrm{HA}$ in group 3 ). HA was prepared by the pharmacy faculty (College of Dental Sciences, Davangere, Karnataka, India), and different HA concentrations were prepared by dissolving 10, 20, and $50 \mathrm{mg}$ of powder into solution (Herb Supply, LLC, Las Vegas, NV, USA). The results demonstrated significant improvements in both the $1 \%$ and $5 \%$ groups, while paradoxically, the $2 \%$ group showed no significant improvement. The initial mean sizes of the triangles were $3.59 \pm 0.93$ (group 1), $3.85 \pm 1.4$ (group 2), and $3.91 \pm 1.81$ in the $5 \%$ group. There were no statistically significant differences between the groups at the start of the study. Six months after injection, the average size in the $1 \%$ group was $3.43 \pm 0.85$ (an increase of $0.16 \mathrm{~mm}$ ) and $4.14 \pm 1.2$ in the $2 \%$ group (a decrease of $-0.29 \mathrm{~mm}$ ). Finally, in the $5 \%$ group, the mean was $3.2 \pm 1.6$, with a significant increase of $0.71 \mathrm{~mm}$ in the interdental papillae. It should be noted that the $5 \%$ group had a smaller relapse from the third and sixth months, unlike the $1 \%$ group, which showed signs of a relapse at 6 months.

Mansouri et al. [15] also found a positive effect of HA on papilla reconstruction in 11 patients (three men and eight women) with a total of 21 interdental papillae in the anterior maxillary region. The authors injected a total of $0.3 \mathrm{~mL}$ of HA gel in three injections.

The result of this study was an average change of $5.04 \pm 1.5 \mathrm{~mm}$ in the papilla defect. At the first follow-up, 3 weeks after the first injection, there was an improvement of $0.17 \pm 0.15$. In the second follow-up, 3 months later, there was an improvement with an average of $1.48 \pm 0.94$. In the third follow-up, 6 months after the injection, the size of the triangle was a further improved, with an average size of $2.38 \pm 1.02$. The total filling of the interdental papillae at 6 months was $2.66 \mathrm{~mm}$. In short, the application of HA was successful in reconstructing the interdental papilla at 6 months. In addition, this was the only article that pointed to a significant association between age and papilla reconstruction $(p<0.01)$. An improvement between $58.72 \pm 20.84 \%$ was observed in patients under 40 years of age, while the percentage of change observed in subjects over 40 years of age was $34.80 \pm 9.55 \%$; however, difference in the amount of change could possibly have been due to the small sample size and dispersion.

The greatest weight in this review with the lower variability was shown by Alhabashneh [16] who presented 21 (14 females, 7 males) patients and a total number of treated interdental papillary sites of 86 . Overall, there was statistically significant difference in black triangle reduction between maxillary and mandibular sites at 3 and 6 months with more reduction observed in maxillary one. On the other hand, the study of $\mathrm{Ni}$ [17] was the only one RCT that concluded the study with a comparison with physiological saline 
solution with no statistically significant differences. The study used a split-mouth design. The test sites were injected with $16 \mathrm{mg} / \mathrm{mL}$ HA gel (Qi Sheng Biological Agent Company Limited, Shanghai, China) at the bases of the deficient papillae. Each site received $0.05-0.1 \mathrm{~mL}$ of solution.

One thing that should be emphasised is the variability in the evaluation methods used in the different studies to evaluate papilla deficiency. For example, Bertl et al. [12] used an intraoral scanner (Tríos TM, 3-Shape, Copenhagen, Denmark) to assess fillings and increase in volume of the papillae [12]. For their research, Lee et al. [13,14] used a standardised photographic device at the outset and in each session in both their studies and analysed the photographs using the program Adobe Photoshop CS5 (Adobe System Inc., San Jose, CA, USA). Singh and Vandana [11] used a modified occlusal stent (made of transparent acrylic resin) to measure interdental papilla deficiency. To evaluate photographs, the authors used ImageJ software (National Institute of Health, Bethesda, MD, USA). Mansouri et al. [15] took a photograph at the start of each session and used the software program ImageJ (National Institute of Health, Bethesda, MD, USA) to calculate the variations in height.

In terms of pain assessment and side effects after HA injection, most articles made little reference [23]. Generally, pain was attributed to the hygroscopic nature of HA, which causes vascular compression and partial occlusion of neighbouring blood vessels. No side effects were reported in any patient within 6 months of the injection.

In the study by Bertl et al. [23], following the initial injection of HA, pain was classified as moderate (VAS: 50-60 points). In contrast, Singh and Vandana [11] used the Numerical Pain Rating Scale (NPRS) to assess patients' discomfort. Post-injection pain was lowest in the $1 \%$ group and highest in the $5 \%$ group. However, the perception of pain decreased in all groups between the first and third injections.

Adverse effects, probably due to the injection, were also reported in three patients. Two patients had severe pain and swelling of the lip after the second injection. In another patient, a painless granuloma of approximately $6 \mathrm{~mm}$ diameter was observed above the mucogingival junction after the first injection. The granuloma persisted for more than 4 weeks.

The advantages of this technique lie in the ease of use, a fast learning curve, and its few side effects (especially in cases of failure where the original defect will not be increased). This cannot be generalised to other surgical techniques. On the other hand, its effectiveness is limited to small defects (less than $1 \mathrm{~mm}$ ).

\subsection{Study Limitations}

We found little consistency in the presentation of results; most of the included studies were of low methodological quality and belonged to the category of case series. In general, there was a lack of blinded randomised studies that could have provided reliable guidance on indications.

Moreover, the small sample sizes of the studies (between 10 and 21 patients) signify that caution should be used when interpreting the results expressed in this meta-analysis. It should be noted that the two studies with the highest weight were conducted by the same author (Lee 2016 JERD [13] and Lee 2016 JPIS [13]).

Finally, consideration should be given to the heterogeneity of the studies. We must accept a hypothesis of lack of homogeneity $(p<0.0009)$ since the degree of heterogeneity was very high $\left(\mathrm{I}^{2}=95 \%\right)$.

\subsection{Future Research Suggestions}

To decrease the heterogeneity of such studies, the inclusion criteria and clinical parameters to be evaluated should be clearly established. We consider that the easiest way to perform future research would be to examine standardised measurements of papilla height. The number of cases should be sufficient to reach an adequate degree of statistical power. 


\section{Conclusions}

The HA injection technique is effective for the reconstruction of interproximal papilla defects within 6 months of injection. The main limitation of the technique is the maximum increase in papilla that can be achieved. This limitation is to be expected with the injection of HA. The average filler height is $0.43 \mathrm{~mm}$, which needs to be maintained with subsequent injections. In addition, total papilla reconstruction is not predictable when the distance between the point of contact and the bone crest is greater than $6 \mathrm{~mm}$ or the area is greater than $0.25 \mathrm{~mm}^{2}$. Regarding the number of injections, there was no consensus in the works studied, although the minimum number of injections was three.

Despite being a minimally invasive and safe technique, certain side effects may occur after the injection of HA, with the most common side effects being moderate pain and swelling of the lip. The appearance of a painless granuloma that disappears after 4 weeks is a very rare side effect.

Although each author has carried out their own treatment protocol, as a general rule the treatment is carried out as follows: the local anaesthetic is injected and then, in each session, a single dose per 0.1-0.2 mL HA was used with a maximum of five applications or until black triangles were no longer visible. The time interval between applications was 3 weeks. No pre-treatment was used except good oral hygiene.

Author Contributions: Conceptualisation: T.R.V.-G. Methodology: A.S.-P., A.J.-G. and C.N.-C. Validation: C.N.-C. Formal analysis: A.S.-P. Investigation: T.R.V.-G. Data curation: T.R.V.-G. and A.S.-P. Writing: T.R.V.-G. and A.S.-P. Review and editing: B.M.-M., C.N.-C., A.J.-G. Visualisation: B.M.-M. and A.J.-G. Supervision: A.S.-P. All authors have read and agreed to the published version of the manuscript.

Funding: This research received no external funding.

Institutional Review Board Statement: Not applicable.

Informed Consent Statement: Not applicable.

Data Availability Statement: The data for this study were obtained from the analysis of published articles.

Acknowledgments: The authors would like to thank J.M.M.-C. and M.J.M.-V. for their invaluable help.

Conflicts of Interest: The authors declare no conflict of interest.

\section{References}

1. Lang, N.P.; Lindhe, J. Clinical Periodontology and Implant Dentistry; John Wiley \& Sons: Chichester, UK, 2015.

2. Kokich, V.O.; Kokich, V.G.; Kiyak, H.A. Perceptions of dental professionals and laypersons to altered dental esthetics: Asymmetric and symmetric situations. Am. J. Orthod. Dentofac. Orthop. 2006, 130, 141-151. [CrossRef] [PubMed]

3. Thomas, M.; Reddy, B.; Reddy, R. Perception differences of altered dental esthetics by dental professionals and laypersons. Indian J. Dent. Res. 2011, 22, 242-247. [CrossRef] [PubMed]

4. Ko-Kimura, N.; Kimura-Hayashi, M.; Yamaguchi, M.; Ikeda, T.; Meguro, D.; Kanekawa, M.; Kasai, K. Some factors associated with open gingival embrasures following orthodontic treatment. Aust. Orthod. J. 2003, 19, 19-24. [PubMed]

5. Seibert, J.; Lindhe, J. Esthetics and periodontal therapy. Textb. Clin. Periodontol. 1989, 2, 477-514.

6. Wu, Y.J.; Tu, Y.K.; Huang, S.M.; Chan, C.P. The influence of the distance from the contact point to the crest of bone on the presence of the interproximal dental papilla. Chang Gung Med. J. 2003, 26, 822-828. [PubMed]

7. Chow, Y.C.; Eber, R.M.; Tsao, Y.P.; Shotwell, J.L.; Wang, H.L. Factors associated with the appearance of gingival papillae. J. Clin. Periodontol. 2010, 37, 719-727. [CrossRef]

8. Sharma, A.A.; Park, J.H. Esthetic considerations in interdental papilla: Remediation and regeneration. J. Esthet. Restor. Dent. 2010, 22, 18-28. [CrossRef]

9. Rohrich, R.J.; Ghavami, A.; Crosby, M.A. The role of hyaluronic acid fillers (restylane) in facial cosmetic surgery: Review and technical considerations. Plast. Reconstr. Surg. 2007, 120, 41S-54S. [CrossRef]

10. Liberati, A.; Altman, D.G.; Tetzlaff, J.; Mulrow, C.; Gotzsche, P.C.; Ioannidis, J.P.A.; Clarke, M.; Devereaux, P.J.; Kleijnen, J.; Moher, D. The PRISMA statement for reporting systematic reviews and meta-analyses of studies that evaluate healthcare interventions: Explanation and elaboration. BMJ 2009, 339, B2700. [CrossRef]

11. Singh, S.; Vandana, K. Use of different concentrations of hyaluronic acid in interdental papillary deficiency treatment: A clinical study. J. Indian Soc. Periodontol. 2019, 23, 35-41. [CrossRef] 
12. Bertl, K.; Gotfredsen, K.; Jensen, S.S.; Bruckmann, C.; Stavropoulos, A. Can hyaluronan injections augment deficient papillae at implant-supported crowns in the anterior maxilla? A randomized controlled clinical trial with 6 months follow-up. Clin. Oral Implant. Res. 2017, 28, 1054-1061. [CrossRef]

13. Lee, W.P.; Kim, H.J.; Yu, S.J.; Kim, B.O. Six month clinical evaluation of interdental papilla reconstruction with injectable hyaluronic acid gel using an image analysis system. J. Esthet. Restor. Dent. 2016, 28, 221-230. [CrossRef] [PubMed]

14. Lee, W.P.; Seo, Y.S.; Kim, H.J.; Yu, S.J.; Kim, B.O. The association between radiographic embrasure morphology and interdental papilla reconstruction using injectable hyaluronic acid gel. J. Periodontal Implant Sci. 2016, 46, 277-287. [CrossRef]

15. Mansouri, S.S.; Ghasemi, M.; Salmani, Z.; Shams, N. Clinical application of hyaluronic acid gel for reconstruction of interdental papilla at the esthetic zone. J. Islam. Dent. Assoc. Iran 2013, 28, 221-230.

16. Alhabashneh, R.; Alomari, S.; Khaleel, B.; Qinawi, H.; Alzaubi, M. Interdental papilla reconstruction using injectable hyaluronic acid: A 6 month prospective longitudinal clinical study. J. Esthet. Restor. Dent. 2021, 33, 531-537. [CrossRef]

17. Ni, J.; Zhong, Z.; Wu, Y.; Shu, R.; Wu, Y.; Li, C. Hyaluronic acid vs. physiological saline for enlarging deficient gingival papillae: A randomized controlled clinical trial and an in vitro study. Ann. Transl. Med. 2021, 9, 759. [CrossRef] [PubMed]

18. Sterne, J.A.C.; Hernán, M.A.; Reeves, B.C.; Savović, J.; Berkman, N.D.; Viswanathan, M.; Henry, D.; Altman, D.G.; Ansari, M.T.; Boutron, I.; et al. ROBINS-I: A tool for assessing risk of bias in non-randomised studies of interventions. BMJ 2016, $355, \mathrm{i} 4919$. [CrossRef]

19. Ziahosseini, P.; Hussain, F.; Millar, B.J. Management of gingival black triangles. Br. Dent. J. 2014, 217, 559-563. [CrossRef]

20. Kurth, J.R.; Kokich, V.G. Open gingival embrasures after orthodontic treatment in adults: Prevalence and etiology. Am. J. Orthod. Dentofac. Orthop. 2001, 120, 116-123. [CrossRef] [PubMed]

21. Choquet, V.; Hermans, M.; Adriaenssens, P.; Daelemans, P.; Tarnow, D.P.; Malevez, C. Clinical and radiographic evaluation of the papilla level adjacent to single-tooth dental implants. A retrospective study in the maxillary anterior region. J. Periodontol. 2001, 72, 1364-1371. [CrossRef] [PubMed]

22. Rodríguez, X.; Vela, X.; Méndez, V.; Segalà, M.; Calvo-Guirado, J.L.; Tarnow, D.P. The effect of abutment dis/reconnections on peri-implant bone resorption: A radiologic study of platform-switched and non-platform-switched implants placed in animals. Clin. Oral Implant. Res. 2013, 24, 305-311. [CrossRef] [PubMed]

23. Salama, H.; Salama, M.A.; Garber, D.; Adar, P. The interproximal height of bone: A guidepost to predictable aesthetic strategies and soft tissue contours in anterior tooth replacement. Pract. Periodontics Aesthet. Dent. 1998, 10, 1131-1141. [PubMed]

24. Roccuzzo, M.; Roccuzzo, A.; Ramanuskaite, A. Papilla height in relation to the distance between bone crest and interproximal contact point at single-tooth implants: A systematic review. Clin. Oral Implant. Res. 2018, 29, 50-61. [CrossRef] [PubMed]

25. Bertl, K.; Gotfredsen, K.; Jensen, S.S.; Bruckmann, C.; Stavropoulos, A. Adverse reaction after hyaluronan injection for minimally invasive papilla volume augmentation. A report on two cases. Clin. Oral Implant. Res. 2017, 28, 871-876. [CrossRef] 